\title{
Opinion Mining of GST Implementation using Supervised Machine Learning Approach
}

\author{
Nandini Tomar \\ Manav Rachna International \\ Institute of Research and Studies \\ (MRIIRS) Haryana, India
}

\author{
Ritesh Srivastava \\ Manav Rachna International \\ Institute of Research and Studies \\ (MRIIRS) Haryana, India
}

\author{
Bindiya Ahuja \\ Manav Rachna International \\ Institute of Research and Studies \\ (MRIIRS) Haryana, India
}

\begin{abstract}
Sentiment Analysis is a way to determine the emotions behind social media discussions. Analyzing social data plays a vital role in knowing people's behavior about an entity or event occurring in the society. Sentiment analysis is widely used in a variety of applications like classifying, summarizing and aggregating reviews from the massive amount of unstructured data that may be available from customer comments, blogs, feedback and reviews on any product or social issue. The Goods \& Service Tax(GST) was debated a lot in the social network as it impacts every citizen of India and there was a state of confusion among people about this amendment in the taxation system. If this state of people can be determined, then it can help in identifying how effectively GST scheme is executing. In this paper, we present Sentiment Analysis (SA) of GST by using the textual content of Twitter to determine the public opinion about GST.
\end{abstract}

\section{Keywords}

Sentiment Analysis, GST, Supervised Machine Learning.

\section{INTRODUCTION}

Now a day internet is flooded with several social network sites like Twitter, Tumblr, Facebook and many more, which provide various ways to connect with other active users worldwide. Twitter [1-4]is one of the popular and vastly used microblogging services on the Internet today. It connects people from diverse areas and helps them to, communicate with each other, participate in online group discussions, share an opinion on a product, service or some social issue in real and quick manner. It enables users to write short text messages, i.e., up to 140 characters long called as "tweets." Twitter provides many interactive features like following, retweet, mentions, etc. that facilitate a way to connect with other like-minded people and this way a group of similar people is formed. The tweets exchanged in such groups can be analyzed to mine opinion of the people about the discussed topic. Millions of people from all over the world are active on Twitter, and this count of active users is increasing every day. So, it becomes a rich source of information and hence a good area of research for many researchers $[5,6]$.

Remaining part of this sections gives a discussion of all related terms we use in this paper.

\subsection{GST in India}

The Goods and Service Tax (GST) bill was passed in the Parliament on 29th March 2017. The Act came into effect on 1st July 2017 and had replaced many Indirect Taxes in India $[2,7]$. The GST is India's most significant tax reform and majorly discussed the topic in online networking groups such as Twitter. Public opinion towards this uniform tax has an essential role in its acceptance.

\subsection{Sentiment Analysis}

The sentiment is an attitude or perception driven by feeling. Sentiment analysis (SA) is the process of determining and measuring the emotional state of response made by opinion [8-11]. SA is a tool that enables companies to analyze what their customers are thinking about their products and services and identifies the trends in the behavior or attitude of their customers toward the products and services they provide concerning their competitors. The Opinion mining of a product is performed by, identifying the sentiments of opinion holder and then classifying their polarity. This work is based on the analysis of Twitter data related to GST to predict people's opinion. Sentiment analysis of Twitter textual data (called tweets) is done with an event and time specific training dataset, which is semi-manually annotated, to capture the sentiments of Indian people towards GST implementation. As our dataset is manually annotated (i.e., labeled as positive or negative) we opt for supervised machine learning approach to perform SA.

Sentiment Analysis can be performed at the following three levels[12, 13]:

(i) Document-level: Sentiment Analysis at this level is used to identify the polarity of a single entity in the given document. Document-level sentiment analysis assumes that each document expresses opinions only on a separate body, e.g., a single product or service and is expressed by a single opinion holder.

(ii) Sentence level: Sentence level classification involves following two steps:

- Subjectivity classification of a sentence into objective or subjective classes.

- Sentiment classification of subjective sentences into positive and negative classes.

(iii) Aspect Level/Feature Level Classification: The goal of this classification is to identify and extract features of the object that have been discussed by the opinion holder and determine the polarity of the opinion

\subsubsection{Techniques of Sentiment Analysis}

Sentiment analysis is widely used in a variety of applications like classifying, summarizing and aggregating reviews from the massive volume of unstructured data that may be available from customer comments, blogs, feedback and reviews on any product or social issue. The SA can be performed by using following two approaches [12]:

(i) Lexicon based Approach

(ii) Machine Learning Approach 
The Lexicon-based Approach relies on a sentiment dictionary, a collection of known and precompiled sentiment terms. The Machine Learning Approach (ML), applies the famous supervised or unsupervised machine learning algorithms and uses linguistic features extracted from the opinion text. Fig. 1 illustrate the different approached that can be used to perform sentiment analysis.

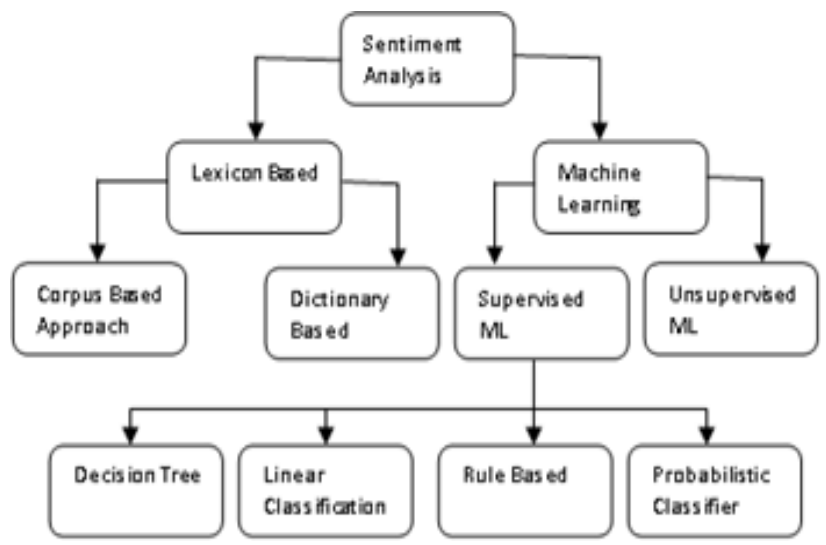

Fig.-1: Sentiment Analysis Techniques

\subsubsection{Lexicon Based Approach}

In lexicon-based approach, pre-built dictionaries of words with associated sentiment orientations is used. This method assumes that the joint polarity of sentences is the sum of individual sentence polarity. Positive Opinion words are used to express some favorable states, while negative opinion words are used to represent some state of disliking. There are also opinion phrases and idioms which together are called opinion lexicon. There are three main approaches to gather the opinion word list. The manual procedure is very time consuming and is usually combined with one of the other two methods. The two automated approaches are:

(i) Dictionary-based approach

(ii) Corpus-based approach.

\section{Dictionary-based Approach:}

Corpus-based methods use a seed set of sentiment words with known polarity and exploit syntactic patterns of co-occurrence patterns to identify new sentiment words and their polarity in a large corpus.

\section{Corpus-based approach}

Using corpus-based approach alone to identify all opinion words, is not as effective as the dictionary-based approach because it is a tedious task to prepare a considerable corpus to cover all words. However, this approach useful in finding domain-specific opinion words and their orientations if a dictionary from only the specific domain is used in the discovery process.

\subsubsection{Machine Learning Approach}

As human learn from past experiences in the same way machines can also be made learned from past experiences, but for machines, the experience is referred to as previous data. Machine Learning is a subset of Artificial Intelligence that teaches computers to learn from previous knowledge. Machine learning algorithms use computational methods to discover information directly from data without relying on a fixed equation as a model. The richness of training data defines the performance of the classifier. Machine learning algorithms find natural patterns in data that generate understanding and help to make better decisions and predictions.

There are following different ways with which a machine can learn:

(i) Supervised Learning: In this approach, learning is achieved by using labeled training data such as spam/not-spam or positive/negative polarity.

(ii) Unsupervised Learning: In this learning method the training data does not include labeled output, and the key task is to find the original forms rather than the mapping.

(iii) Semi-supervised Learning: It is a combination of supervised and unsupervised learning. In this method, learning is performed with training data which is a mixture of labeled and unlabeled examples.

(iv) Reinforcement learning: It is a goal-oriented learning method based on interaction with the environment. It is built on the reward system, and the rewards are attained from the sequence of action.

This paper is organized into seven sections. In next section-2 a literature review is given defining the related work done by different authors in the area of Sentiment Analysis. Section-3 define the experimental setup required to execute this work. In section-4, we define proposed approach and system architecture. Further section- 5 give detailed discussion on result obtained with each model. Next, section-6 conclude this work along with the scope of improvement and future work. Finally, section-7 provide the references used in this work.

\section{LITERATURE REVIEW}

The growth in social media networks has made sentiment analysis a popular research area, in recent years. Sentiment plays a crucial role in the decision-making process. In the field of information retrieval, web analysis, decision making, and product/service reviews opinion mining has become increasingly important.

In [14] unsupervised method is used to perform classification. To determine the semantic orientation of a phrase two seed words are used, i.e., 'poor' \& 'excellent' and a pointwise mutual information method was used. [15]covers techniques and approaches that promise to enable opinion-oriented information-seeking systems directly. The material on summarization of evaluative text and broader issues regarding privacy, manipulation, and economic impact that the development of opinion-oriented information-access services gives rise are included in this paper. In [16] part of speech(POS) patterns are used to extract the sentiment from given phase, and unknown sentiment phrase is used as a query term. The top $\mathrm{n}$ number of relevant phrases are extracted, and the sentiment analysis is done on them by using the lexicon of nearby known relevant phrases. An Automatic Seed Word Selection method [17] was proposed for unsupervised sentiment classification of product reviews in Chinese. The lexical-based and machine learning-based approaches are combined in [13] with the aim of introducing a hybrid architecture with higher accuracy than the pure lexical method and provides more structure and increased redundancy than machine learning approach. The Senti-lexical algorithm [9] to find the polarity of a review as positive, negative or neutral is proposed to handle words which have negation effect on the 
reviews. The role of emoticons is also discussed in this paper. In [18] a fuzzy inference system is designed based on experimentally developed fuzzy membership functions and concepts of hedges to standardized and formulate the process of strength quantification of subjective sentences when the strength of opinion word get modified by the presence of ngram adverbial modifiers pattern in the sentence. Srivastava, R., \& Bhatia [19]has performed the real-time analysis of the tweets related to a targeted event (e.g., election) to identify those potential sub-events that occurred in the real world, discussed over Twitter and cause the significant change in the aggregated sentiment score of the targeted event with time. Such type of analysis can enrich the real-time decisionmaking ability of the event bearer. The proposed approach utilizes a three-step process: (1) Real-time sentiment analysis of tweets (2) Application of Bayesian Change Points Detection to determine the sentiment change points (3) Major sub-events detection that has influenced the sentiments of targeted events.

\section{EXPERIMENTAL SETUP}

The execution of this work is performed on a machine with core i3, 2.4GHz, $3 \mathrm{MB} \mathrm{L} 3$ cache processor with $4 \mathrm{~GB}$ of RAM. We perform training and testing of the machine learning classifier, Support Vector Machine (SVM)[20-22], with a powerful machine learning tool WEKA[23,24]. Tweets are extracted from Twitter open API [25] by executing a script written in R language. Various R tools like 'twitteR' [26]and 'tm' [27] package are installed to obtain tweets from Twitter.

\subsection{SVM}

We classify GST related tweets in positive and negative classes using SVM, a machine learning classifier. SVM is a powerful supervised machine learning algorithm that can be applied to both classification or regression-type problems. In SVM two data-points, called support vectors, from each of the two groups of the data set are chosen, in such a way that the distance from that point to a line drawn separating the class is maximum or halfway between them. In this model, the machine tries to learn from the most likely positive or negative tweets. The least likely ones are chosen as support vectors.

- In binary class classification to decide which class a new data point will be in, SVM views it as a pdimensional vector (a list of $\mathrm{p}$ numbers), and we want to know whether we can separate such points with a p-1-dimensional hyperplane. Binary classification is called a Linear Classifier.

- SVMs can also efficiently perform a Non-linear Classification using what is called the kernel trick, implicitly mapping their inputs into highdimensional feature spaces.

Support Vector Machine algorithm first finds a hyperplane that best divides given data set into binary classes, as shown in the following Fig. 2.

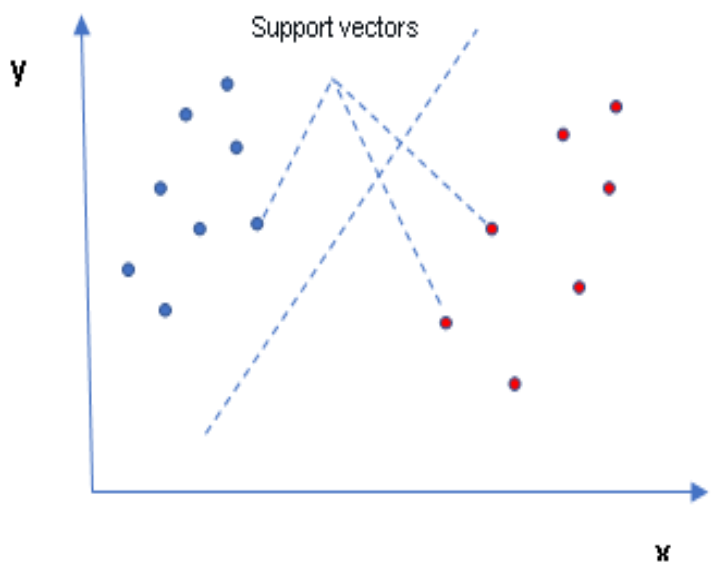

Fig.-2: Two-Class Classification

Support vectors are those data points that are nearest to the defined hyperplane. The points of a data set that, if removed, would alter the position of the dividing hyperplane and considered as the critical elements of a data set.

The SVM's algorithm first starts learning from data that has already been classified, which is represented in numerical labels with each number representing a category. SVM then groups the data with the same label in each convex hull. From there, it determines where the hyperplane is by calculating the connecting points between the convex hull. Once SVM identifies the points that are nearest to each other, it computes the hyperplane, that is a plane separating the labels.

\subsection{WEKA}

Implementation of this work is done using a powerful machine learning tool WEKA. WEKA[24] is an open source analysis program shared by the machine learning group at the University of Waikato in New Zealand. It is a data mining software with a set of machine learning algorithms that can be applied to a data-set directly or called from own Java code. Weka contains tools for data pre-processing, classification, regression, clustering, association rules, and visualization [23]. Weka is a collection of tools for:

$$
\begin{array}{ll}
\text { - } & \text { Regression } \\
\text { - } & \text { Clustering } \\
\text { - Association } \\
\text { - } \\
\text { - } \text { Clata pre-processification }
\end{array}
$$

\section{PROPOSED APPROACH}

Fig-3 depicts all the steps to be executed for sentiment analysis. The proposed approach of this work is divided into following steps:

(i) Data Collection \& Training Data Preparation

(ii) Preprocessing of Collected Data

(iii) Train \& Test the Classifier

\subsection{Data Collection \& Training Data Preparation}

GST related tweets are extracted from twitter open API. On retrieved tweets, subjectivity test is done with the help of SENTWORDNET[28] dictionary which is a publicly available resource for opinion mining. Finally, Sentiment 
analysis is done using popular machine learning tool WEKA. Weka is a data mining software that uses a collection of machine learning algorithms. These algorithms can be applied directly to the data or called from the Java code.

As we are using supervised machine learning approach whose performance highly relies upon its training dataset, use of precise and accurate training data is essential. So, we have constructed training dataset by encapsulating data from two sources:

(i) IMDb [1],[29] Movie Reviews

(ii) Manually Annotated GST Tweets

- We have used IMDb movie reviews, which is highly polar and will make training data set rich in st vocabulary for positive and negative sentiment words. It includes 1000 positive and 1000 negative reviews.

GST related tweets are extracted from Twitter open API. We have extracted post-GST implementation tweets of the period of one month, i.e., from $1^{\text {st }}$ October 2017 to 30 October 2017. On collected tweets, manual annotation is done along with subjectivity test. Subjectivity test is, finding out the text that holds the feeling or sentiment about a discussed topic which can either be positive, negative or neutral. To perform this task, we use opinion word list from SENTIWORDNET.

Manually annotated tweets are specific to GST and include some domain specific terms. GST is abbreviated as Gabbar Singh Tax, Genuine Simple Tax, Great Selfish Tax, etc., some other terms such as antimoneyday, a tool to loot and transforming infrastructure, etc. are also used for GST that shows opinion (positive or negative) of Indian population towards GST. We manually annotated around 2000 tweets (1000 positive and 1000 negative) from extracted GST related tweets. The selection of the tweets is made uniform across the period of data collection. We finally get 865 tweets with positive polarity and 972 tweets with negative polarity. To balance the data from both the negative and positive class, we select 800 positives and 800 negative polarity tweets.

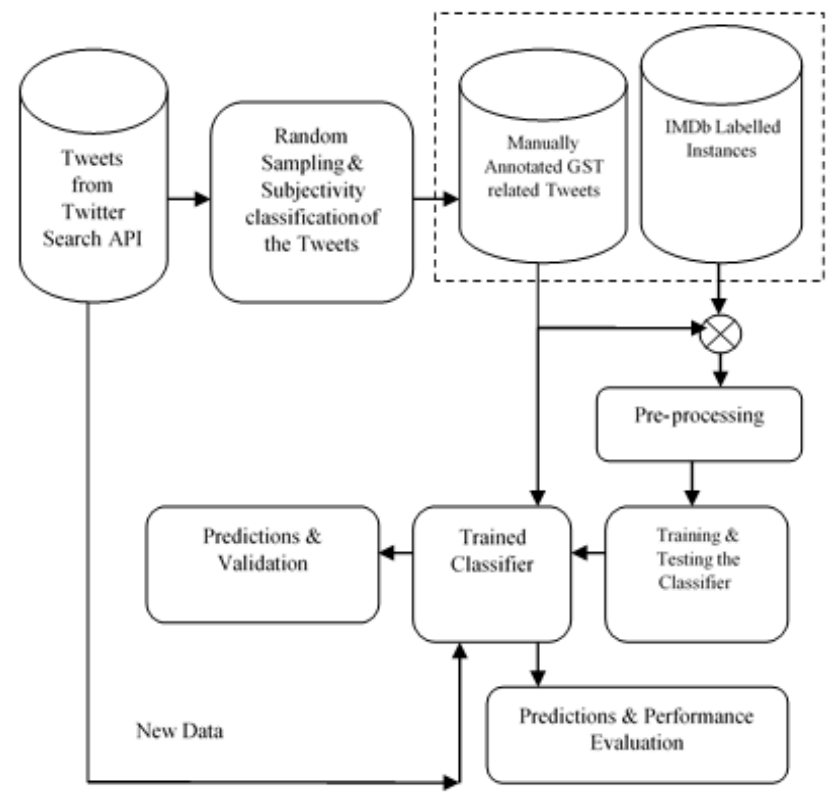

Fig.-3: Proposed Approach

\subsection{Preprocessing of Collected Data}

Pre-processing is the essential step to remove the noisy data. The pre-processing module filters data obtained from the given dataset. The collected dataset may contain some nonsentiment words such as symbols, links, abbreviations, stop words and some twitter specific terms such as \#tags, @tags, name, URL's, retweets, etc. called as noise that needs to be removed before applying it to the classifier to improve classifiers efficiency.

Pre-processing steps includes the following:
(i) Tokenization
(ii) Normalization
(iii) Stemming

\subsubsection{Tokenization}

Tokenization process breaks a text stream into individual words, phrases or other meaningful elements called as tokens. Tokenization will convert tweets in training dataset into word vector. After that normalization will be performed on collected word vector.

\subsubsection{Normalization}

Normalization process includes the following steps:

(i) Eliminating stop words (e.g., a the, at, for, etc.) and names

(ii) Eliminating \# tags, @tags and URL's

(iii) Elimination of special symbols and numbers

(iv) Case sensitivity

\subsubsection{Stemming}

The goal of stemming is to reduce related forms of a word to a common base form. Stemming algorithm breaks the word into small stem by inspecting words from both the sides and then stem word from suffix side. E.g., car, cars, car's, cars', all these words will be replaced with a single base word car after executing the stemming algorithm.

\subsection{Train \& Test the Classifier}

Next step after preprocessing is training and testing the classifier on given dataset. We use a different combination of training and test data to perform training and testing of SVM classifier. This way we define two different models which are as follows:

\subsubsection{Model-1}

In this model, we use IMDb movie review to train the classifier and twitter data(related to GST) to test the classifier.

\subsubsection{Model-2}

In this model, we train and test the SVM classifier with encapsulated data (i.e., IMDb + Domain specific Twitter data) using $\mathrm{k}$-fold $(\mathrm{k}=10)$ cross-validation.

\section{RESULT ANALYSIS AND DISCUSSION}

This section presents result analysis of all three models we experimented in this work. The performance of the classification model is evaluated by using performance measures, i.e., precision, recall, F1-score, ROC curve, and accuracy[30]. These performance measures are calculated with the help of confusion matrix. 


\subsection{Confusion Matrix Obtained with Each Model}

A confusion matrix is a table that is used to describe the performance of a classification model on a set of test data for which the actual values are known[31].

\subsubsection{Confusion Matrix Obtained with Model-1}

With model-2 we achieve $63.12 \%$ of accuracy. Table-1 shows the statistical values obtained with model-1 as a confusion matrix with calculated accuracy.

Table 1. Confusion Matrix Obtained with Model-1

\begin{tabular}{|c|c|c|c|c|}
\hline \multirow{2}{*}{\multicolumn{2}{|c|}{ 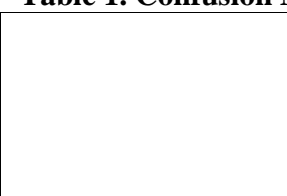 }} & \multicolumn{2}{|c|}{ Predicted } & \multirow{2}{*}{$\begin{array}{l}\text { The } \\
\text { accuracy of } \\
\text { the } \\
\text { classifier } \\
\text { (in \%) }\end{array}$} \\
\hline & & $\begin{array}{l}\text { Positive } \\
\text { Tweets }\end{array}$ & $\begin{array}{l}\text { Negative } \\
\text { Tweets }\end{array}$ & \\
\hline \multirow{3}{*}{ Actual } & $\begin{array}{l}\text { Positive } \\
\text { Instances }\end{array}$ & $\begin{array}{l}493 \\
(\mathrm{TP})\end{array}$ & $\begin{array}{l}307 \\
(\mathrm{FN})\end{array}$ & 61.62 \\
\hline & $\begin{array}{l}\text { Negative } \\
\text { Instances }\end{array}$ & $\begin{array}{l}288 \\
(\mathrm{FP})\end{array}$ & $\begin{array}{l}512 \\
(\mathrm{TN})\end{array}$ & 64 \\
\hline & & 781 & 819 & 62.81 \\
\hline
\end{tabular}

\subsubsection{Confusion Matrix Obtained with Model-2}

Model-2 is our key model in which we perform classification with the encapsulated dataset. We attain expected enhanced accuracy of $73.28 \%$ with this model. Table-2 shows the confusion matrix obtained with model-2.

Table 2. Confusion Matrix Obtained with Model-2

\begin{tabular}{|c|c|c|c|c|}
\hline & \multicolumn{2}{|c|}{ Predicted } & \multirow{2}{*}{$\begin{array}{l}\text { The } \\
\text { accuracy of } \\
\text { the } \\
\text { classifier } \\
(\text { in \%) }\end{array}$} \\
\hline & & $\begin{array}{l}\text { Positive } \\
\text { Tweets }\end{array}$ & $\begin{array}{l}\text { Negative } \\
\text { Tweets }\end{array}$ & \\
\hline \multirow{3}{*}{ Actual } & $\begin{array}{l}\text { Positive } \\
\text { Instances }\end{array}$ & $\begin{array}{l}1326 \\
(\mathrm{TP})\end{array}$ & $\begin{array}{l}474 \\
(\mathrm{FN})\end{array}$ & 73.67 \\
\hline & $\begin{array}{l}\text { Negative } \\
\text { Instances }\end{array}$ & $\begin{array}{l}488 \\
(\mathrm{FP})\end{array}$ & $\begin{array}{l}1312 \\
(\mathrm{TN})\end{array}$ & 72.89 \\
\hline & & 1814 & 1786 & 73.28 \\
\hline
\end{tabular}

\subsection{Performance Analysis of Each Model:}

The below-mentioned Table- 3 shows the Precision, Recall, FMeasure and the Accuracy obtained with both proposed model. Accuracy is the percentage of all correct predicted instances against all predicted instances[32]. With model-2 we attain expected improved accuracy of $73.28 \%$. We believe that the classifier's performance improved by making training data more precise and rich in sentiment vocabulary.
Table 3. Performance Statistics obtained with Model-1 \& Model-2

\begin{tabular}{|l|l|l|}
\hline & Model-1 & Model-2 \\
\hline $\begin{array}{l}\text { Model } \\
\text { Explanation }\end{array}$ & $\begin{array}{l}\text { The model trained } \\
\text { on IMDb movie } \\
\text { reviews and tested } \\
\text { over collected } \\
\text { tweets related to } \\
\text { GST }\end{array}$ & $\begin{array}{l}\text { The model trained } \\
\text { and tested on } \\
\text { summed up data } \\
\text { (i.e., IMDb + } \\
\text { collected GS } \\
\text { related tweets) }\end{array}$ \\
\hline Precision & 63.12 & 73.09 \\
\hline Recall & 61.62 & 73.67 \\
\hline F-Measure & 62.36 & 73.28 \\
\hline Accuracy & 62.81 & \\
\hline
\end{tabular}

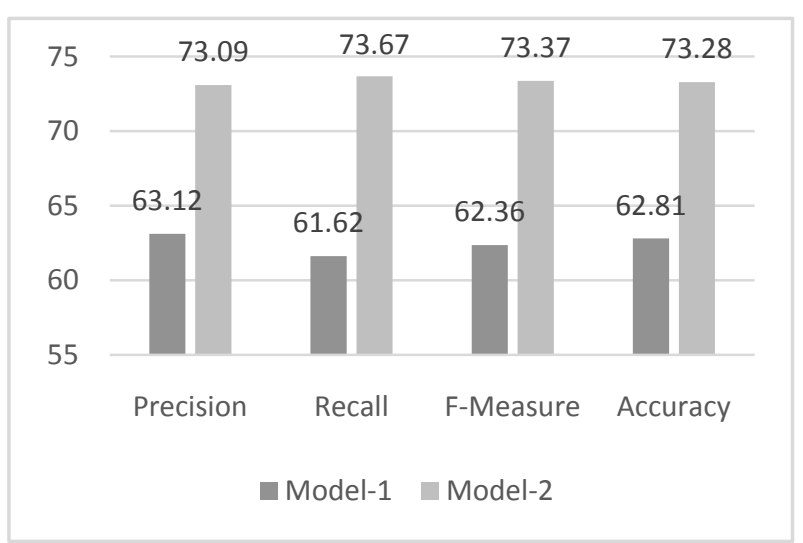

Fig-4: Comparison of Performance Measures Obtained with Model-1 \& Model-2

From Fig-4 we can analyze that the performance of the classifier gets improved with $73.28 \%$ when we train and test the classifier with encapsulated (IMDb+Domain Specific Data) data set.

\section{CONCLUSIONS AND FUTURE WORK}

The Goods \& Service Tax(GST) was debated a lot in the social network as it impacts every citizen of India and there was a state of confusion among people about this amendment in the taxation system. If this state of people can be determined, then it can help in identifying how effectively GST scheme is executing. In this paper, we present Sentiment Analysis (SA) of GST by using the textual content of Twitter to determine the public opinion about GST. We have successfully created a classifier that can perform binary classification on the Twitter textual data (tweets) related to GST implementation with $73.28 \%$ accuracy. We incorporated the domain \& time specific features to our training dataset by encapsulating manually annotated GST related tweets with the labeled reviews of IMDb dataset. This way we attain improved accuracy as compared to the model-1 trained only on IMDb.

In this work, we build a classifier to perform only binary classification (i.e., positive and negative polarity). However, during manual annotation of GST related tweets, we observe 
that many people show a state of confusion about GST. Therefore, in future, we will perform multiclass classification by adding one more class of confusion. Moreover, the accuracy of the classifier can further be improved by adding more filtering process in preprocessing of the training dataset.

\section{REFERENCES}

[1] R. Srivastava, H. Kumar, M. Bhatia, and S. Jain, "Analyzing Delhi assembly election 2015 using textual content of social network," in Proceedings of the Sixth International Conference on Computer and Communication Technology 2015, 2015, pp. 78-85: ACM.

[2] K. Pabreja, "GST sentiment analysis using twitter data," IJAR, vol. 3, no. 7, pp. 660-662, 2017.

[3] Z. Z. Alp and Ş. G. Öğüdücü, "Identifying topical influencers on twitter based on user behavior and network topology," Knowledge-Based Systems, vol. 141, pp. 211-221, 2018.

[4] I. Anger and C. Kittl, "Measuring influence on Twitter," in Proceedings of the 11th International Conference on Knowledge Management and Knowledge Technologies, 2011, p. 31: ACM.

[5] D. A. Shamma, L. Kennedy, and E. F. Churchill, "Tweet the debates: understanding community annotation of uncollected sources," in Proceedings of the first SIGMM workshop on Social media, 2009, pp. 3-10: ACM.

[6] H. Kwak, C. Lee, H. Park, and S. Moon, "What is Twitter, a social network or a news media?," in Proceedings of the 19th international conference on World wide web, 2010, pp. 591-600: ACM.

[7] (27-04-2018). Goods and Services Tax (India). Available:

https://en.wikipedia.org/wiki/Goods_and_Services_Tax_ (India)

[8] V. Mittal and I. Kashyap, "Empirical Study of Impact of Various Concept Drifts in Data Stream Mining Methods," International Journal of Intelligent Systems and Applications, vol. 8, no. 12, p. 65, 2016.

[9] D. Mumtaz and B. Ahuja, "Sentiment analysis of movie review data using Senti-Lexicon Algorithm," in Applied and Theoretical Computing and Communication Technology (iCATccT), 2016 2nd International Conference on, 2016, pp. 592-597: IEEE.

[10] R. Srivastava and M. Bhatia, "Offline vs. Online Sentiment Analysis: Issues With Sentiment Analysis of Online Micro-Texts," International Journal of Information Retrieval Research (IJIRR), vol. 7, no. 4, pp. $1-18,2017$.

[11] R. Srivastava, M. Bhatia, H. K. Srivastava, and C. Sahu, "Exploiting grammatical dependencies for fine-grained opinion mining," in Computer and communication technology (iccct), 2010 international conference on, 2010, pp. 768-775: IEEE.

[12] V. B. Vaghela and B. M. Jadav, "Analysis of various sentiment classification techniques," International Journal of Computer Applications, vol. 140, no. 3, 2016.

[13] D. Mumtaz and B. Ahuja, "A Lexical and Machine Learning-Based Hybrid System for Sentiment Analysis," in Innovations in Computational Intelligence: Springer, 2018, pp. 165-175.

[14] P. D. Turney, "Thumbs up or thumbs down?: semantic orientation applied to unsupervised classification of reviews," in Proceedings of the 40th annual meeting on association for computational linguistics, 2002, pp. 417 424: Association for Computational Linguistics.

[15] B. Pang and L. Lee, "Opinion mining and sentiment analysis," Foundations and Trends ${ }^{\circledR}$ in Information Retrieval, vol. 2, no. 1-2, pp. 1-135, 2008.

[16] T.-C. Peng and C.-C. Shih, "Using Chinese part-ofspeech patterns for sentiment phrase identification and opinion extraction in user generated reviews," in Digital Information Management (ICDIM), 2010 Fifth International Conference on, 2010, pp. 120-127: IEEE.

[17] T. Zagibalov and J. Carroll, "Unsupervised classification of sentiment and objectivity in Chinese text," in Proceedings of the Third International Joint Conference on Natural Language Processing: Volume-I, 2008.

[18] R. Srivastava and M. Bhatia, "Quantifying modified opinion strength: A fuzzy inference system for sentiment analysis," in Advances in Computing, Communications and Informatics (ICACCI), 2013 International Conference on, 2013, pp. 1512-1519: IEEE.

[19] R. Srivastava and M. Bhatia, "Real-Time Unspecified Major Sub-Events Detection in the Twitter Data Stream That Cause the Change in the Sentiment Score of the Targeted Event," International Journal of Information Technology and Web Engineering (IJITWE), vol. 12, no. 4, pp. 1-21, 2017.

[20] F. Sebastiani, "Machine learning in automated text categorization," ACM computing surveys (CSUR), vol. 34, no. 1, pp. 1-47, 2002.

[21] Hsu, Chih-Wei, Chih-Chung Chang, and Chih-Jen Lin. "A practical guide to support vector classification." (2003): 1-16.

[22] Joachims, Thorsten. "Text categorization with support vector machines: Learning with many relevant features." European conference on machine learning. Springer, Berlin, Heidelberg, 1998.

[23] M. Hall, E. Frank, G. Holmes, B. Pfahringer, P. Reutemann, and I. H. Witten, "The WEKA data mining software: an update," ACM SIGKDD explorations newsletter, vol. 11, no. 1, pp. 10-18, 2009.

[24] Holmes, Geoffrey, Andrew Donkin, and Ian H. Witten. "Weka: A machine learning workbench." In Intelligent Information Systems, 1994. Proceedings of the 1994 Second Australian and New Zealand Conference on, pp. 357-361. IEEE, 1994.

[25] https://developer.twitter.com. (22-04-2018). Docs.

[26] Gentry, Jeff, Maintainer Jeff Gentry, Suggests RSQLite, and RMySQL License Artistic. "Package 'twitteR'." R package version 1, no. 9 (2016)

[27] I. Feinerer, "Introduction to the tm Package Text Mining in $\mathrm{R}, "$ ed, 2017.

[28] S. Baccianella, A. Esuli, and F. Sebastiani, "Sentiwordnet 3.0: an enhanced lexical resource for 
sentiment analysis and opinion mining," in LREC, 2010, vol. 10 , no. 2010 , pp. $2200-2204$.

[29] B. Pang and L. Lee, "A sentimental education: Sentiment analysis using subjectivity summarization based on minimum cuts," in Proceedings of the 42nd annual meeting on Association for Computational Linguistics, 2004, p. 271: Association for Computational Linguistics.

[30] Forman, George. "An extensive empirical study of feature selection metrics for text classification." Journal of machine learning research 3, no. Mar (2003): 12891305.

[31] Accuracy, Precision, Recall \& F1 Score: Interpretation Of ... http://blog.exsilio.com/all/accuracy-precisionrecall-f1-score-interpretation-of (accessed June 04, 2018).

[32] R. Sharma, S. Nigam, and R. Jain, "Determination of Polarity of sentences using Sentiment Orientation System," International journal of Advances in Computer Science and Technology (IJACST) WARSE, vol. 3, no. 3, pp. 182-187, 2014. 